This is a post-peer-review, pre-copyedit version of an article published in Mediterranean journal of mathematics. The final authenticated version is available online at: http://dx.doi.org/10.1007/s00009-020-01688-z

\title{
Caterpillars are Antimagic
}

\author{
Antoni Lozano* $\quad$ Mercè Mora $^{\dagger} \quad$ Carlos Seara $^{\ddagger} \quad$ Joaquín Tey $^{\S}$
}

口

\begin{abstract}
An antimagic labeling of a graph $G$ is a bijection from the set of edges $E(G)$ to $\{1,2, \ldots,|E(G)|\}$ such that all vertex sums are pairwise distinct, where the vertex sum at vertex $u$ is the sum of the labels assigned to the edges incident to $u$. A graph is called antimagic when it has an antimagic labeling. Hartsfield and Ringel conjectured that every simple connected graph other than $K_{2}$ is antimagic and the conjecture remains open even for trees. Here we prove that caterpillars are antimagic by means of an $O(n \log n)$ algorithm.
\end{abstract}

\section{Introduction}

All graphs considered in this paper are finite, undirected, connected, and simple. Given a graph $G$, we denote its set of vertices by $V(G)$ and its set of edges by $E(G)$. For every vertex $v \in V(G)$, we denote by $E_{G}(v)$ the set of edges incident to $v$ in $G$. The degree of a vertex $v \in V(G)$ is $d_{G}(v)=\left|E_{G}(v)\right|$ (we will just write $d(v)$ when $G$ is clear from context). For undefined terminology about basic graph theory we refer the reader to [4].

An (edge) labeling of a graph $G$ is an injection from $E(G)$ to the set of nonnegative integers. A labeling of $G$ is called antimagic if it is a bijection $\phi: E(G) \rightarrow\{1,2, \ldots,|E(G)|\}$ such that all vertex sums are pairwise distinct, where the vertex sum at vertex $u$ is $\sum_{e \in E_{G}(u)} \phi(e)$. A graph is called antimagic if it has an antimagic labeling. The following conjecture from Hartsfield and Ringel [13] is well known.

Conjecture 1. [13] Every connected graph other than $K_{2}$ is antimagic.

Classes of graphs which are known to be antimagic include: paths, stars, complete graphs, cycles, wheels, and complete bipartite graphs $K_{2, m}$ [13]; graphs of order $n$ with maximum degree at least $n-3$ [24]; dense graphs (i.e., graphs having minimum degree $\Omega(\log n))$ and complete partite graphs but $K_{2}$ [2, 11]; toroidal grid graphs [22]; lattice grids

\footnotetext{
${ }^{*}$ Computer Science Department, Universitat Politècnica de Catalunya, Spain, antoni@cs.upc.edu

${ }^{\dagger}$ Mathematics Department, Universitat Politècnica de Catalunya, Spain, merce.mora@upc.edu

${ }^{\ddagger}$ Mathematics Department, Universitat Politècnica de Catalunya, Spain, carlos.seara@upc.edu

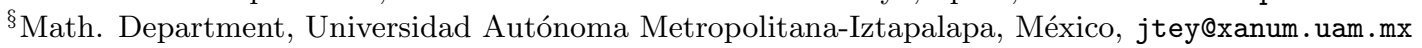

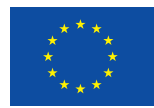

This project has received funding from the European Union's Horizon 2020 research and innovation programme under the Marie Skłodowska-Curie grant agreement No 734922 . 
and prisms [6]; regular bipartite graphs [8]; odd degree regular graphs [9]; even degree regular graphs [5]; cubic graphs [21]; generalized pyramid graphs [3]; graph products [23]; and Cartesian product of graphs [7, 25] (see the dynamic survey [12] for more details on antimagic labelings). However, the conjecture is still open for the general class of trees.

Conjecture 2. [13] Every tree other than $K_{2}$ is antimagic.

One of the best known results for trees is due to Kaplan, Lev, and Roditty [16], who proved that any tree having more than two vertices and at most one vertex of degree two is antimagic (see also [20]). In this paper we focus on caterpillars, that is, trees of order at least 3 such that the removal of their leaves produces a path. In [19] the authors give sufficient conditions for a caterpillar to be antimagic and, recently, it has been shown that that caterpillars with maximum degree 3 are antimagic [10]. In this paper we take a step further proving that every caterpillar is antimagic.

Theorem 1. Caterpillars are antimagic. Furthermore, there exists an algorithm that, given a caterpillar $C$ of order $n$, produces an antimagic labeling for $C$ in time $O(n \log n)$.

Concretely, in Section 2 we provide the above mentioned algorithm, whose correctness and time bound are shown in Section 3. Therefore, our method follows a constructive approach, in contrast with the use of the Combinatorial NullStellenSatz method [1, 14, 17, which is the regular technique used in several of the references above.

\section{Construction of an Antimagic Labeling}

Suppose that $C$ is a caterpillar with $m$ edges and let $P=\left(v_{0}, \ldots, v_{p}\right)$ be a longest path in $C$. Edges in $P$ will be called pathedges, while edges not in $P$ will be called legs. We use the notation $e_{i}=\left\{v_{i-1}, v_{i}\right\}(1 \leq i \leq p)$ for pathedges and $f_{i}$ for the leg incident to a vertex $v_{i}$ of degree 3. We define $E_{0}=\left\{e_{i}: i \equiv p(\bmod 2)\right\}$ and $E_{1}=\left\{e_{i}: i \not \equiv p(\bmod 2)\right\}$, so that $\left\{E_{0}, E_{1}\right\}$ is a partition of the set of pathedges such that $e_{p} \in E_{0}$ and any two incident edges of $P$ belong to different sets. The size of these sets is $\left|E_{0}\right|=\lceil p / 2\rceil$ and $\left|E_{1}\right|=\lfloor p / 2\rfloor$.

Let $[a, b]=\{n \in \mathbb{N}: a \leq n \leq b\}$ and $[a, \infty)=\{n \in \mathbb{N}: n \geq a\}$, where $a$ and $b$ are positive integers. Split the set of available labels $L=[1, m]$ into the two subsets $L_{0}=[1, m-\lfloor p / 2\rfloor]$ and $L_{1}=[m-\lfloor p / 2\rfloor+1, m]$ of sizes $m-\lfloor p / 2\rfloor$ and $\lfloor p / 2\rfloor$, respectively.

We now describe an algorithm in three steps to construct a labeling $\phi$ of $C$ that will be shown to be antimagic in Section 3 (see the pseudocode in Page 4 and an example in Figure 1). Given a vertex $u$ in $C$ and a labeling $\phi$ of $C$, we denote the vertex sum at $u$ by $s(u)$, that is, $s(u)=\sum_{e \in E_{C}(u)} \phi(e)$. When we describe the construction of the labeling, we also use the notation $s(u)$ to refer to a partial sum at $u$, that is, the sum of the values $\phi(e)$ for all edges $e$ incident to $u$ for which a label has already been assigned at that step of the algorithm. Similarly, we use $\phi(E(C))$ to denote the set of labels used up to that step.

In the first step, a label is assigned to all pathedges and a few legs incident to vertices of degree 3. Roughly speaking, the algorithm alternatively assigns consecutive labels from 
the lists $L_{0}$ and $L_{1}$ to the pathedges with some exceptions, and taking into account that the label of edge $e_{p}$ must belong to $L_{0}$. Concretely, the edges of $E_{1}$ receive labels from $L_{1}$ in increasing order, and the edges of $E_{0}$ receive labels from $L_{0}$ in increasing order with the following exception. When assigning a label to the edge $e_{i} \in E_{0}, 1<i \leq p$, we check if the following condition holds:

$\boldsymbol{Q}(i): \phi\left(e_{i-1}\right)=\phi\left(e_{k}\right)+\phi\left(e_{k+1}\right)$ for some $k \in\{1, \ldots, i-3\}$ such that $d\left(v_{k}\right)=3$.

If $Q(i)$ does not hold, then we assign the next unused label of $L_{0}$ to $e_{i}$. Otherwise, we assign the next unused label of $L_{0}$ to the leg $f_{k}$ and the next one, to $e_{i}$. Such a vertex $v_{k}$ of degree 3 , whose leg $f_{k}$ has been labeled at this step, will be called light vertex, while the rest of vertices of degree at least three will be called heavy vertices. The set of all light vertices obtained when finishing this step is denoted by $U_{p}$, while the set of heavy vertices is denoted by $W$.

In the second step, for each heavy vertex $v$, we randomly assign unused labels from $L_{0}$ to all but one of the legs incident to $v$.

Finally, it only remains to label one leg for each heavy vertex. To do that, in the third step we list the remaining legs in increasing order of the partial vertex sums at their corresponding incident heavy vertices. Then, we sort the remaining labels in $L_{0}$ in increasing order and assign them to the previous list of legs in the same order.
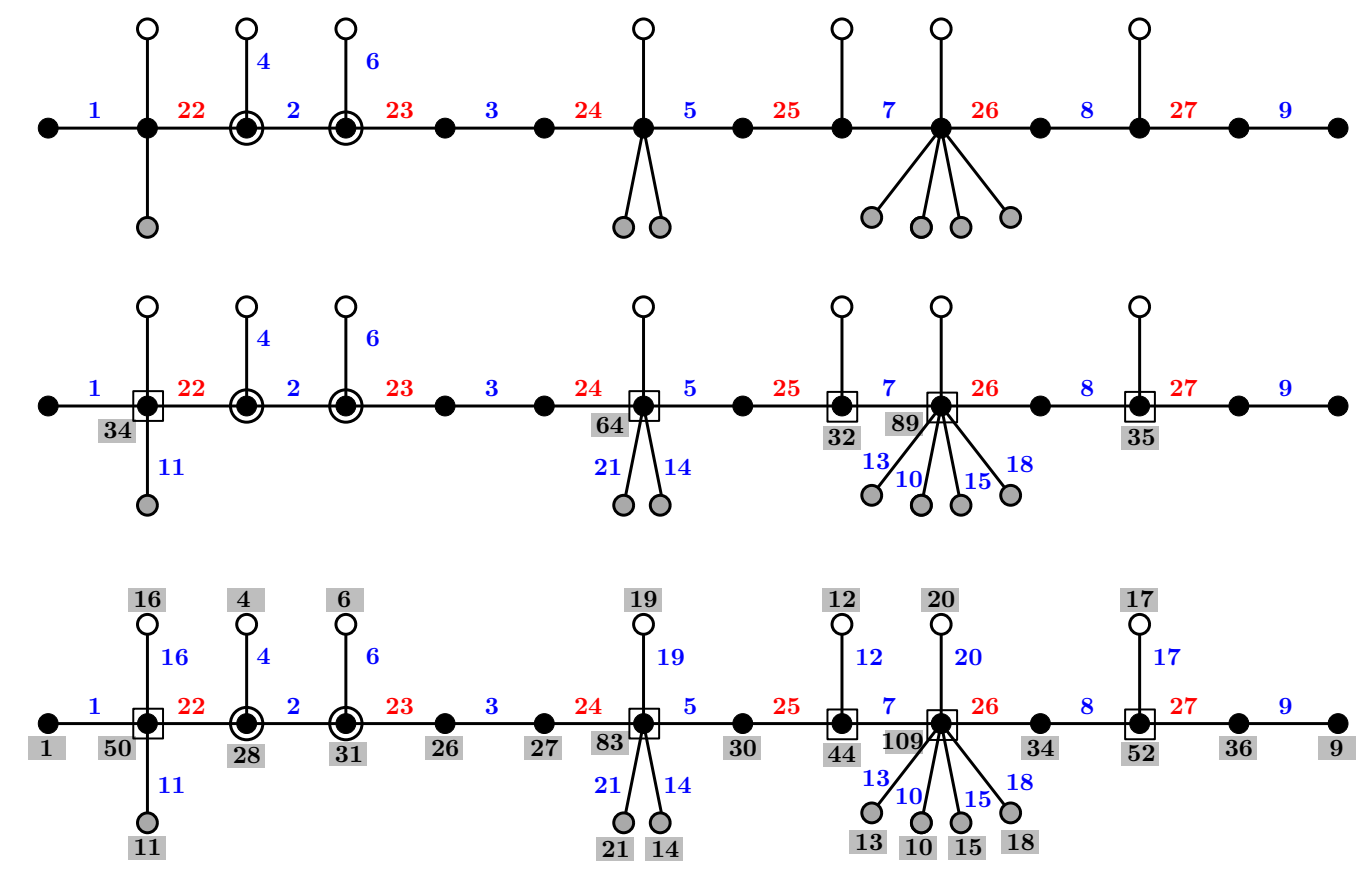

Figure 1: Example of a labeling of a caterpillar with a longest path of odd length. In this case, $L_{0}=[1,21]$ and $L_{2}=[22,27]$. Top, Step 1: Labeling the pathedges and legs incident to light vertices (circled vertices). Middle, Step 2: Labeling all legs but one for each heavy vertex (squared vertices). Bottom, Step 3: Labeling the last leg for each heavy vertex. 


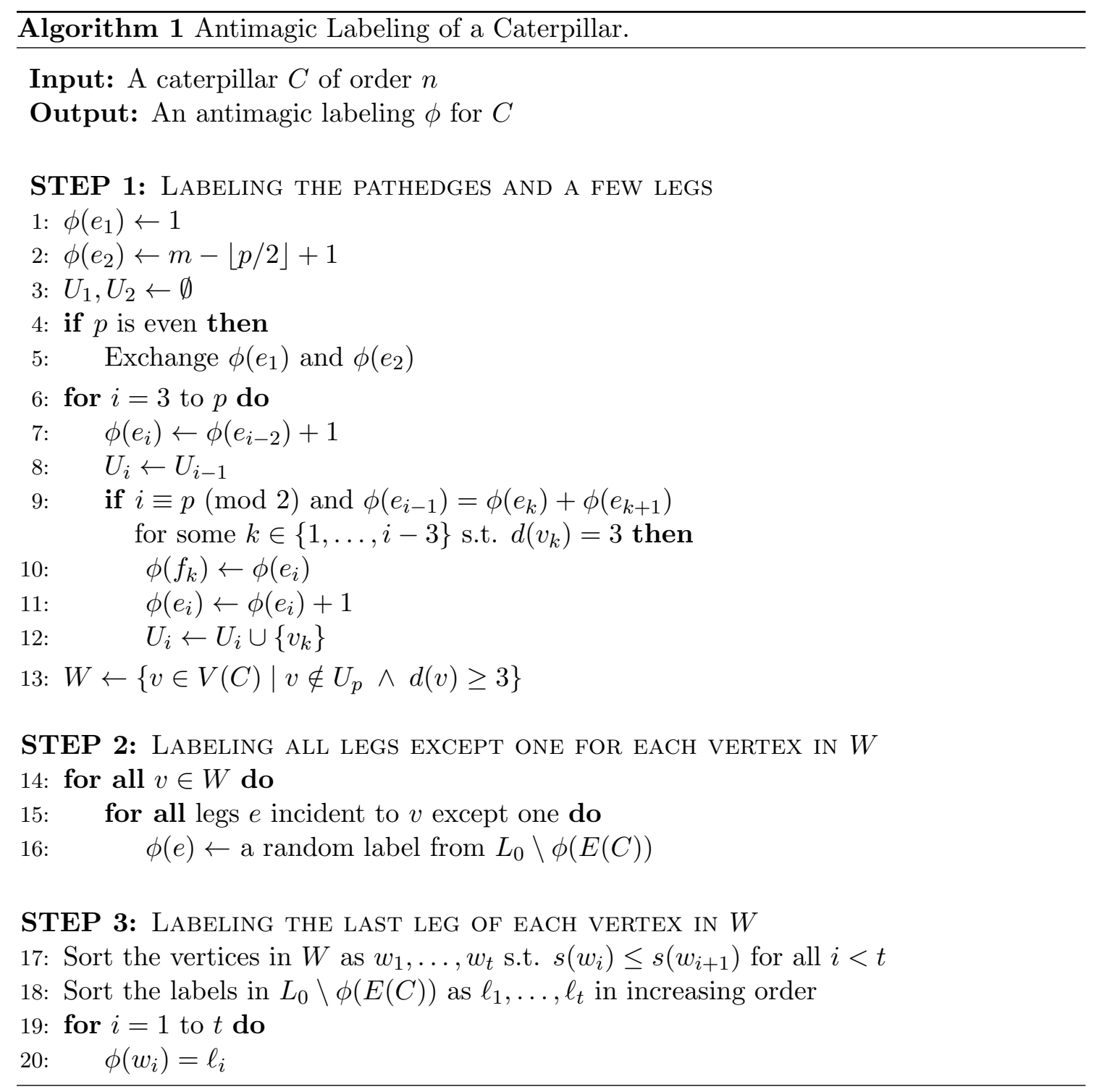

\section{Proof of Theorem 1}

In this section we prove that the labeling produced by Algorithm 1 for a caterpillar of order $n$ is antimagic and can be found in time $O(n \log n)$, thus proving Theorem 1 . The following lemmas will be used in the proofs of correctness and efficiency of the algorithm.

We first introduce some notation. We denote by $F_{0}$ the set of legs incident to light vertices. Recall that the set $U_{i}$ introduced in the algorithm contains light vertices, that is, vertices of degree 3 whose legs have been labeled up to iteration $i$ of the for loop in Step 1. Hence, $\left|F_{0}\right|=\left|U_{p}\right|$. Let $E_{2}$ be the set of edges not belonging to $E_{0} \cup E_{1} \cup F_{0}$, that is, $\left\{E_{0}, E_{1}, E_{2}, F_{0}\right\}$ is a partition of the edge set $E(C)$. 
Lemma 1. For every $i \in\{1,2, \ldots, p\}$ we have

$$
\phi\left(e_{i}\right)= \begin{cases}\left\lceil\frac{i}{2}\right\rceil+\left|U_{i}\right|, & \text { if } e_{i} \in E_{0} ; \\ m-\left\lfloor\frac{p}{2}\right\rfloor+\left\lceil\frac{i}{2}\right\rceil, & \text { if } e_{i} \in E_{1} .\end{cases}
$$

Moreover,

(1) $\phi\left(E_{0} \cup F_{0}\right)=\left[1,\lceil p / 2\rceil+\left|U_{p}\right|\right] \subseteq L_{0}$;

(2) $\phi\left(E_{1}\right)=L_{1}=[m-\lfloor p / 2\rfloor+1, m]$;

(3) $\phi\left(E_{2}\right)=\left[\lceil p / 2\rceil+\left|U_{p}\right|+1, m-\lfloor p / 2\rfloor\right] \subseteq L_{0}$.

Proof. First we check that the values $\phi\left(e_{1}\right)$ and $\phi\left(e_{2}\right)$ defined in Equation 1 are correct. Indeed, if $p$ is odd, then $e_{1} \in E_{0}, e_{2} \in E_{1}$, and the labels assigned after the execution of lines $1-5$ of the algorithm are $\phi\left(e_{1}\right)=1=\lceil 1 / 2\rceil+\left|U_{1}\right|$ and $\phi\left(e_{2}\right)=m-\lfloor p / 2\rfloor+1=$ $m-\lfloor p / 2\rfloor+\lceil 2 / 2\rceil$. If $p$ is even, then $e_{1} \in E_{1}, e_{2} \in E_{0}$, and the labels assigned in lines $1-5$ are $\phi\left(e_{1}\right)=m-\lfloor p / 2\rfloor+1=m-\lfloor p / 2\rfloor+\lceil 1 / 2\rceil$ and $\phi\left(e_{2}\right)=1=\lceil 2 / 2\rceil+\left|U_{2}\right|$.

Now, we show that the value $\phi\left(e_{i}\right), 3 \leq i \leq p$, defined in Equation 1 corresponds to the same value calculated in the for loop at lines 6-12. Suppose that $3 \leq i \leq p$ and all values given in Equation 11 up to $\phi\left(e_{i-1}\right)$ are correct. Then, either $e_{i} \in E_{0}$ or $e_{i} \in E_{1}$. Suppose first that $e_{i} \in E_{1}$. Then, lines 10-12 are not executed and $\phi\left(e_{i}\right)=\phi\left(e_{i-2}\right)+1$. Since $e_{i-2} \in E_{1}$ and, by hypothesis, $\phi\left(e_{i-2}\right)=m-\lfloor p / 2\rfloor+\left\lceil\frac{i-2}{2}\right\rceil$, we have

$$
\phi\left(e_{i}\right)=m-\left\lfloor\frac{p}{2}\right\rfloor+\left\lceil\frac{i-2}{2}\right\rceil+1=m-\left\lfloor\frac{p}{2}\right\rfloor+\left\lceil\frac{i-2}{2}+1\right\rceil=m-\left\lfloor\frac{p}{2}\right\rfloor+\left\lceil\frac{i}{2}\right\rceil .
$$

Now suppose that $e_{i} \in E_{0}$. In this case, $e_{i-2} \in E_{0}$ and, by hypothesis, $\phi\left(e_{i-2}\right)=\left\lceil\frac{i-2}{2}\right\rceil+$ $\left|U_{i-2}\right|$. Note that $e_{i-1} \in E_{1}$ and, then, the iteration for $i-1$ produces $U_{i-1}=U_{i-2}$. If condition $Q(i)$ does not hold, then lines 10-12 are not executed. Thus, $\left|U_{i}\right|=\left|U_{i-1}\right|$ and $\phi\left(e_{i}\right)=\phi\left(e_{i-2}\right)+1$, and we get

$$
\phi\left(e_{i}\right)=\left\lceil\frac{i-2}{2}\right\rceil+\left|U_{i-2}\right|+1=\left\lceil\frac{i-2}{2}+1\right\rceil+\left|U_{i-2}\right|=\left\lceil\frac{i}{2}\right\rceil+\left|U_{i}\right| .
$$

Otherwise, after the execution of lines 10-12 we have that $\phi\left(e_{i}\right)=\phi\left(e_{i-2}\right)+2$ and $\left|U_{i}\right|=$ $\left|U_{i-1}\right|+1$. Since $e_{i-2} \in E_{0}$ and, by hypothesis, $\phi\left(e_{i-2}\right)=\left\lceil\frac{i-2}{2}\right\rceil+\left|U_{i-2}\right|$, we obtain

$$
\phi\left(e_{i}\right)=\left\lceil\frac{i-2}{2}\right\rceil+\left|U_{i-2}\right|+2=\left\lceil\frac{i-2}{2}+1\right\rceil+\left|U_{i-2}\right|+1=\left\lceil\frac{i}{2}\right\rceil+\left|U_{i}\right| .
$$

In both of the above cases, the value $\phi\left(e_{i}\right)$ computed by the algorithm coincides with that defined in Equation 1.

Now, we prove the first item. On the one hand, the values obtained for the edges in $E_{0}$ range between 1 and $\lceil p / 2\rceil+\left|U_{p}\right|$ by Equation 1 and the value of a leg $f_{k} \in F_{0}$ (i.e., a leg incident to a light vertex) is less than the value of some edge belonging to $E_{0}$. Hence, $\phi\left(E_{0} \cup F_{0}\right) \subseteq\left[1,\lceil p / 2\rceil+\left|U_{p}\right|\right]$. Since $\phi$ is an injection and $\left|E_{0} \cup F_{0}\right|=\lceil p / 2\rceil+\left|U_{p}\right|$, we 
have $\phi\left(E_{0} \cup F_{0}\right)=\left[1,\lceil p / 2\rceil+\left|U_{p}\right|\right]$. On the other hand, $\left|E_{0} \cup F_{0}\right| \leq m-\left|E_{1}\right|=m-\lfloor p / 2\rfloor$. Thus, $\phi\left(E_{0} \cup F_{0}\right)=\left[1,\lceil p / 2\rceil+\left|U_{p}\right|\right] \subseteq L_{0}$.

As for the second item, since $\left|E_{1}\right|=\lfloor p / 2\rfloor=\left|L_{1}\right|, \phi\left(E_{1}\right) \subseteq L_{1}$ and $\phi$ is an injection, we have that $\phi\left(E_{1}\right)=L_{1}=[m-\lfloor p / 2\rfloor+1, m]$. Finally, in Steps 2 and 3, the algorithm assigns the remaining labels to the remaining edges. Therefore, $\phi\left(E_{2}\right)=[1, m] \backslash \phi\left(E_{0} \cup F_{0} \cup E_{1}\right)=$ $\left[\lceil p / 2\rceil+\left|U_{p}\right|+1, m-\lfloor p / 2\rfloor\right] \subseteq L_{0}$ and the third item is true.

Lemma 2. For any $i, j$ such that $1 \leq i \leq j<p$, we have:

(1) $m-\lfloor p / 2\rfloor+2 \leq \phi\left(e_{i}\right)+\phi\left(e_{i+1}\right) \leq m+\lceil p / 2\rceil+\left|U_{p}\right|$;

(2) If $i<j$, then $\phi\left(e_{i}\right)+\phi\left(e_{i+1}\right)<\phi\left(e_{j}\right)+\phi\left(e_{j+1}\right)$;

(3) If $v_{i}$ and $v_{j}$ are light vertices and $i<j$, then $\phi\left(f_{i}\right)<\phi\left(f_{j}\right)$.

Proof. We begin by proving the first item. Since one of the edges from $\left\{e_{i}, e_{i+1}\right\}$ belongs to $E_{0}$ and the other one to $E_{1}$, according to Equation 1 in Lemma 1 , we have

$$
\phi\left(e_{i}\right)+\phi\left(e_{i+1}\right)=m-\left\lfloor\frac{p}{2}\right\rfloor+\left\lceil\frac{i}{2}\right\rceil+\left\lceil\frac{i+1}{2}\right\rceil+h,
$$

for some $h, 0 \leq h \leq\left|U_{p}\right|$. Hence, the lower bound trivially holds. For the upper bound, note that

$$
m-\left\lfloor\frac{p}{2}\right\rfloor+\left\lceil\frac{i}{2}\right\rceil+\left\lceil\frac{i+1}{2}\right\rceil+h \leq m-\left\lfloor\frac{p}{2}\right\rfloor+\left\lceil\frac{p-1}{2}\right\rceil+\left\lceil\frac{p}{2}\right\rceil+\left|U_{p}\right| \leq m+\left\lceil\frac{p}{2}\right\rceil+\left|U_{p}\right| .
$$

To prove the second item, let $g:[1, p-1] \rightarrow \mathbb{N}$ be the function

$$
g(i)=\phi\left(e_{i}\right)+\phi\left(e_{i+1}\right) .
$$

Now, it is enough to show that function $g$ is strictly increasing. Note that for any $i$ such that $1 \leq i \leq p-2$, proving $g(i)<g(i+1)$ is equivalent to proving the inequality:

$$
\phi\left(e_{i}\right)<\phi\left(e_{i+2}\right)
$$

whenever $1 \leq i \leq p-2$. Hence, we consider two cases according to the value of $\phi$ for pathedges given in Equation 1. If $e_{i} \in E_{0}$, then

$$
\phi\left(e_{i}\right)<\phi\left(e_{i+2}\right) \Leftrightarrow\left\lceil\frac{i}{2}\right\rceil+\left|U_{i}\right|<\left\lceil\frac{i+2}{2}\right\rceil+\left|U_{i+2}\right| \Leftrightarrow\left|U_{i}\right|<1+\left|U_{i+2}\right|,
$$

which is true. Now, if $e_{i} \in E_{1}$, then

$$
\phi\left(e_{i}\right)<\phi\left(e_{i+2}\right) \Leftrightarrow m-\left\lfloor\frac{p}{2}\right\rfloor+\left\lceil\frac{i}{2}\right\rceil<m-\left\lfloor\frac{p}{2}\right\rfloor+\left\lceil\frac{i+2}{2}\right\rceil \Leftrightarrow\left\lceil\frac{i}{2}\right\rceil<\left\lceil\frac{i+2}{2}\right\rceil,
$$

which is also true. This concludes the proof of the second item.

Let us now prove the third item. Suppose that $1 \leq i<j \leq p-1$. Notice that labels $\phi\left(f_{i}\right)$ and $\phi\left(f_{j}\right)$ are assigned in the first step of the algorithm if $Q\left(i^{\prime}\right)$ and $Q\left(j^{\prime}\right)$ 
hold when assigning the label of some pathedges $e_{i^{\prime}}$ and $e_{j^{\prime}}$ of $E_{0}$, with $i^{\prime}>i$ and $j^{\prime}>j$, and such that $\phi\left(e_{i^{\prime}-1}\right)=\phi\left(e_{i}\right)+\phi\left(e_{i+1}\right)$ and $\phi\left(e_{j^{\prime}-1}\right)=\phi\left(e_{j}\right)+\phi\left(e_{j+1}\right)$. On the one hand, since $i<j$, we deduce by the preceding item that $\phi\left(e_{i^{\prime}-1}\right)<\phi\left(e_{j^{\prime}-1}\right)$. Besides, $e_{i^{\prime}-1}, e_{j^{\prime}-1} \in E_{1}$, and from Inequality 2 we deduce $i^{\prime}<j^{\prime}$. On the other hand, the labels assigned to the edges $f_{i}$ and $f_{j}$ are $\phi\left(f_{i}\right)=\phi\left(e_{i^{\prime}-2}\right)+1$ and $\phi\left(f_{j}\right)=\phi\left(e_{j^{\prime}-2}\right)+1$, where $e_{i^{\prime}-2}, e_{j^{\prime}-2} \in E_{0}$ and $i^{\prime}-2<j^{\prime}-2$. Again, from Inequality 2 , we conclude that $\phi\left(f_{i}\right)=\phi\left(e_{i^{\prime}-2}\right)+1<\phi\left(e_{j^{\prime}-2}\right)+1=\phi\left(f_{j}\right)$.

\subsection{Proof of Correctness}

Now we show the correctness of the algorithm, that is, we show that, given a caterpillar $C$, Algorithm 1 produces an antimagic labeling of $C$.

Since $E(C)=E_{0} \cup E_{1} \cup E_{2} \cup F_{0}$, we have by Lemma 1 that $\phi$ is a bijection from $E(C)$ onto $[1, m]$. To prove that $\phi$ is an antimagic labeling, it remains to show that all vertex sums are pairwise different.

Consider the partition $\left\{V_{1}, V_{2}, V_{3}\right\}$ of $V(C)$, where $V_{1}$ is the set of vertices of degree 1 , $V_{2}$ is the set of vertices of degree 2 and light vertices, and $V_{3}$ is the set of heavy vertices. Let $s\left(V_{i}\right)=\left\{s(v): v \in V_{i}\right\}$, for $1 \leq i \leq 3$. It is enough to prove that for each $i, 1 \leq i \leq 3$, all sums in $s\left(V_{i}\right)$ are pairwise distinct and the sets $s\left(V_{1}\right), s\left(V_{2}\right), s\left(V_{3}\right)$ are pairwise disjoint.

We begin by checking that the vertices of $V_{1}$ have pairwise distinct vertex sums. The vertex sum at a vertex of degree one is the value of the label of the pendant edge incident to it. Since all edges receive different labels, vertex sums at vertices of degree one are pairwise distinct.

Now let us establish an interval of possible values in $s\left(V_{1}\right)$. Notice that a pendant edge is either a leg, or the first or the last edge of the path $P$. According to Lemma 1, legs are always labeled with elements from $L_{0}$. Regarding the first and last edges of the path $P$, if $p$ is odd, their labels are from $L_{0}$, whereas if $p$ is even, these pathedges are labeled with the smallest values of $L_{1}$ and $L_{0}$, respectively, that is, $m-\lfloor p / 2\rfloor+1$ and 1 . Hence, $s\left(V_{1}\right) \subseteq L_{0} \cup\{m-\lfloor p / 2\rfloor+1\}=[1, m-\lfloor p / 2\rfloor+1]$.

In order to check that all vertices of $V_{2}$ have pairwise distinct sums, consider two distinct vertices $v_{j}, v_{k} \in V_{2}$. Now, we distinguish three cases. In the first case, suppose that $v_{j}$ and $v_{k}$ are vertices of degree 2 . If $1 \leq j<k<p$, then their sums can be expressed as

$$
s\left(v_{j}\right)=\phi\left(e_{j}\right)+\phi\left(e_{j+1}\right), \quad s\left(v_{k}\right)=\phi\left(e_{k}\right)+\phi\left(e_{k+1}\right),
$$

and by Lemma $2, s\left(v_{j}\right)<s\left(v_{k}\right)$. In the second case, suppose that both $v_{j}$ and $v_{k}$ are light vertices. If $1 \leq j<k<p$, then, similarly to the first case, their sums are

$$
s\left(v_{j}\right)=\phi\left(e_{j}\right)+\phi\left(e_{j+1}\right)+\phi\left(f_{j}\right), \quad s\left(v_{k}\right)=\phi\left(e_{k}\right)+\phi\left(e_{k+1}\right)+\phi\left(f_{k}\right)
$$

and, by Lemma 2 we conclude that $s\left(v_{j}\right)<s\left(v_{k}\right)$. In the third case, suppose that one of the vertices, say $v_{j}$, has degree 2 and the other one, that is $v_{k}$, is a light vertex. In such a case, we know that $Q(i)$ holds for some $i$, with $k+2<i<p$, that is,

$$
\phi\left(e_{k}\right)+\phi\left(e_{k+1}\right)=\phi\left(e_{i-1}\right), \phi\left(f_{k}\right)=\phi\left(e_{i-2}\right)+1, \text { and } \phi\left(e_{i}\right)=\phi\left(e_{i-2}\right)+2 .
$$


Besides,

$$
s\left(v_{k}\right)=\phi\left(e_{k}\right)+\phi\left(e_{k+1}\right)+\phi\left(f_{k}\right) \text { and } s\left(v_{j}\right)=\phi\left(e_{j}\right)+\phi\left(e_{j+1}\right) .
$$

Now, by applying Lemma 2 , if $j \leq k$, then

$$
\begin{aligned}
s\left(v_{j}\right) & =\phi\left(e_{j}\right)+\phi\left(e_{j+1}\right) \leq \phi\left(e_{k}\right)+\phi\left(e_{k+1}\right) \\
& <\phi\left(e_{k}\right)+\phi\left(e_{k+1}\right)+\phi\left(f_{k}\right)=s\left(v_{k}\right) ;
\end{aligned}
$$

if $k<j \leq i-2$, then

$$
\begin{aligned}
s\left(v_{j}\right) & =\phi\left(e_{j}\right)+\phi\left(e_{j+1}\right) \leq \phi\left(e_{i-2}\right)+\phi\left(e_{i-1}\right) \\
& <\phi\left(e_{i-1}\right)+\phi\left(e_{i-2}\right)+1 \\
& =\phi\left(e_{k}\right)+\phi\left(e_{k+1}\right)+\phi\left(f_{k}\right)=s\left(v_{k}\right) ;
\end{aligned}
$$

and if $k<i-1 \leq j$, then

$$
\begin{aligned}
s\left(v_{j}\right) & =\phi\left(e_{j}\right)+\phi\left(e_{j+1}\right) \geq \phi\left(e_{i-1}\right)+\phi\left(e_{i}\right) \\
& =\phi\left(e_{i-1}\right)+\phi\left(e_{i-2}\right)+2 \\
& =\phi\left(e_{k}\right)+\phi\left(e_{k+1}\right)+\phi\left(f_{k}\right)+1>s\left(v_{k}\right) .
\end{aligned}
$$

Hence, all vertices in $V_{2}$ have different vertex sums.

We now determine the interval of possible values in $s\left(V_{2}\right)$. Let $v_{k} \in V_{2}$. For the lower bound, we have $s\left(v_{k}\right) \geq \phi\left(e_{k}\right)+\phi\left(e_{k+1}\right) \geq m-\lfloor p / 2\rfloor+2$ by Lemma 2. For the upper bound, again by Lemma 2, the vertex sum at any vertex of degree two can be bounded by $m+\lceil p / 2\rceil+\left|U_{p}\right|$, while if $v_{k}$ is a light vertex, then for some $i$ with $k+2<i \leq p$ and $i \equiv p(\bmod 2)$ we have:

$s\left(v_{k}\right)=\phi\left(e_{k}\right)+\phi\left(e_{k+1}\right)+\phi\left(f_{k}\right)=\phi\left(e_{i-1}\right)+\phi\left(f_{k}\right)=\phi\left(e_{i-1}\right)+\phi\left(e_{i-2}\right)+1<m+\left\lceil\frac{p}{2}\right\rceil+\left|U_{p}\right|+1$.

Therefore, $s\left(V_{2}\right) \subseteq\left[m-\lfloor p / 2\rfloor+2, m+\lceil p / 2\rceil+\left|U_{p}\right|\right]$.

Finally, recall that $V_{3}$ is the set of heavy vertices. Due to the way labels are assigned in Steps 2 and 3, all vertex sums of heavy vertices will be pairwise distinct.

Let us calculate the interval of possible values of their vertex sums. Let $v_{i}$ be a heavy vertex and suppose, in the first place, that $d\left(v_{i}\right)=3$. Since, by construction, the leg of $v_{i}$ has not been labeled in Step 1, the partial sum of $v_{i}$ after Step 1 must be at least $m+1$. Indeed, suppose on the contrary that $\phi\left(e_{i}\right)+\phi\left(e_{i+1}\right) \leq m$. By Lemma 2, we would have $\phi\left(e_{i}\right)+\phi\left(e_{i+1}\right)=\ell \in[m-\lfloor p / 2\rfloor+2, m] \subseteq L_{1}$, so that after assigning the label $\ell$ to some edge $e_{j-1}$ of $E_{1}$ in Step 1, condition $Q(j)$ would have held for some $j$, with $j>i$, implying that the leg incident to $v_{i}$ would have been labeled in Step 1, a contradiction. Hence, $\phi\left(e_{i}\right)+\phi\left(e_{i+1}\right) \geq m+1$. By Lemma 1, the labels for its leg are at least $\lceil p / 2\rceil+\left|U_{p}\right|+1$. Therefore,

$$
s\left(v_{i}\right) \geq m+\left\lceil\frac{p}{2}\right\rceil+\left|U_{p}\right|+2 .
$$


Suppose now that $d\left(v_{i}\right) \geq 4$. Then, since there are at least two legs incident with $v_{i}$, we have

$$
\begin{aligned}
s\left(v_{i}\right) & \geq \phi\left(e_{i}\right)+\phi\left(e_{i+1}\right)+\left(\left\lceil\frac{p}{2}\right\rceil+\left|U_{p}\right|+1\right)+\left(\left\lceil\frac{p}{2}\right\rceil+\left|U_{p}\right|+2\right) \\
& \geq m-\left\lfloor\frac{p}{2}\right\rfloor+2+2 \cdot\left\lceil\frac{p}{2}\right\rceil+2\left|U_{p}\right|+3 \geq m+\left\lceil\frac{p}{2}\right\rceil+2\left|U_{p}\right|+5 .
\end{aligned}
$$

Hence, $s\left(V_{3}\right) \subseteq\left[m+\lceil p / 2\rceil+\left|U_{p}\right|+2, \infty\right)$.

Notice that $s\left(V_{1}\right), s\left(V_{2}\right)$, and $s\left(V_{3}\right)$ have been shown to be included in pairwise disjoint intervals and, as a consequence, they must also be pairwise disjoint.

\subsection{Proof of Efficiency}

Finally, we show that Algorithm 1 runs in time $O(n \log n)$.

Assignments in Step 1 of the algorithm can be done in constant time, except for the one at line 13, which requires computing a set difference and can be done in time $O(m)$. Condition in line 9 (which is equivalent to the fact that both $e_{i} \in E_{0}$ and $Q(i)$ hold) can be checked in linear time globally due to the fact that partial sums are increasing as variable $i$ increases, as shown in Lemma 2. Therefore, the cost of Step 1 is the result of the linear loop at lines 6-12 and the assignment at line 13, that is, $O(m)$. Step 2 visits at most $m$ edges and assigns a random label to each of them, but labels can be chosen increasingly from the unused labels in $L_{0}$, thus giving a cost $O(m)$. Step 3 requires time $O(m \log m)$ due to fact that the partial vertex sums must be sorted (line 17).

The total cost of the algorithm is, then, $O(m \log m)$, but since $C$ is a tree, $n=m+1$ and the cost can be expressed as $O(n \log n)$.

Theorem 1 now follows from the proofs of correctness and efficiency contained, respectively, in Subsections 3.1 and 3.2 .

\section{Conclusions and Open Problems}

We consider a consequence of our main result regarding oriented graphs. Define a labeling of a directed graph $D$ with $m$ arcs as a bijection from the set of $\operatorname{arcs}$ of $D$ to $[1, m]$. A labeling of $D$ is said to be antimagic if all oriented vertex sums are pairwise distinct, where the oriented vertex sum of a vertex in $D$ is the sum of labels of all incoming arcs minus that of all outgoing arcs. A graph is said to have an antimagic orientation if it has an orientation which admits an antimagic labeling. Hefetz, Mütze, and Schwartz [15] formulate the following conjecture.

Conjecture 3. [15] Every connected graph admits an antimagic orientation.

As the authors point out in the paper, every bipartite antimagic undirected graph $G$ admits an antimagic orientation by simply orienting all edges in the same direction between the two stable sets of $G$. Therefore, we can derive the following corollary from Theorem 1, which was originally proved in [18]. 
Corollary 1. [18] Caterpillars have antimagic orientations.

Lobsters, defined as trees such that the removal of their leaves produces a caterpillar, are a natural class of trees to which one can try to apply the techniques presented in this paper. A first question in the line of the above corollary would be the following.

Open problem 1. Do lobsters have antimagic orientations?

More generally, we state the following question.

Open problem 2. Are lobsters antimagic?

\section{Acknowledgments}

Antoni Lozano is supported by the European Research Council (ERC) under the European Union's Horizon 2020 research and innovation programme (grant agreement ERC2014-CoG 648276 AUTAR). Mercè Mora is supported by projects Gen. Cat. DGR 2017SGR1336, MINECO MTM2015-63791-R, and H2020-MSCA-RISE project 734922CONNECT. Carlos Seara is supported by projects Gen. Cat. DGR 2017SGR1640, MINECO MTM2015-63791-R, and H2020-MSCA-RISE project 734922-CONNECT. Joaquín Tey is supported by project PRODEP-12612731.

\section{References}

[1] N. Alon. Combinatorial Nullstellensatz. Combinatorics, Probability and Computing, 8, (1999), 7-29.

[2] N. Alon, G. Kaplan, A. Lev, Y. Roditty, and R. Yuster. Dense graphs are antimagic. Journal of Graph Theory, 47(4), (2004), 297-309.

[3] S. Arumugam, M. Miller, O. Phanalasy, and J. Ryan. Antimagic labeling of generalized pyramid graphs. Acta Mathematica Sinica, 30(2), (2014), 283-290.

[4] G. Chartrand, L. Lesniak, and P. Zhang. Graphs and Digraphs, fifth edition. CRC Press, Boca Raton, 2011.

[5] F. Chang, Y.-Ch. Liang, Z. Pan, and X. Zhu. Antimagic labeling of regular graphs. Journal of Graph Theory, 82(4), (2016), 339-349.

[6] Y. Cheng. Lattice grids and prisms are antimagic. Theoretical Computer Science, $374(1-3),(2007), 66-73$.

[7] Y. Cheng. A new class of antimagic Cartesian product graphs. Discrete Mathematics, 308(24), (2008), 6441-6448.

[8] D. W. Cranston. Regular bipartite graphs are antimagic. Journal of Graph Theory, 60(3), (2009), 173-182. 
[9] D. W. Cranston, Y.-Ch. Liang, and X. Zhu. Regular graphs of odd degree are antimagic. Journal of Graph Theory, 80(1), (2015), 28-33.

[10] K. Deng and Y. Li. Caterpillars with maximum degree 3 are antimagic. Discrete Mathematics, 342, (2019), 1799-1801

[11] T. Eccles. Graphs of large linear size are antimagic. Journal of Graph Theory, 81(3), (2016), 236-261.

[12] J. A. Gallian. A dynamic survey of graph labeling. The Electronic Journal of Combinatorics, 5, (2018), \#DS6.

[13] N. Hartsfield and G. Ringel. Pearls in Graph Theory: A Comprehensive Introduction. Academic Press, INC., Boston, 1990 (revised version, 1994), 108-109.

[14] D. Hefetz. Antimagic graphs via the Combinatorial NullStellenSatz. Journal of Graph Theory, 50(4), (2005), 263-272.

[15] D. Hefetz, T. Mütze, and J. Schwartz. On antimagic directed graphs. Journal of Graph Theory, 64 (2010), 219-232.

[16] G. Kaplan, A. Lev, and Y. Roditty. On zero-sum partitions and antimagic trees. Discrete Mathematics, 309, (2009), 2010-2014.

[17] A. Lladó and M. Miller. Approximate results for rainbow labelings. Periodica Mathematica Hungarica, 74(1), (2017), 11-21.

[18] A. Lozano. Caterpillars Have Antimagic Orientations. An. St. Univ. Ovidius Constanta Ser. Mat., 26(3), (2018), 171-180.

[19] A. Lozano, M. Mora, and C. Seara. Antimagic labelings of caterpillars. Applied Mathematics and Computation, 347, (2019), 734-740.

[20] Y.-Ch. Liang, T.-L. Wong, and X. Zhu. Antimagic labeling of trees. Discrete Mathematics, 331, (2014), 9-14.

[21] Y.-Ch. Liang and X. Zhu. Antimagic labeling of cubic graphs. Journal of Graph Theory, 75 (1), (2014), 31-36.

[22] T.-M. Wang. Toroidal grids are antimagic. Proc. 11th Annual International Computing and Combinatorics Conference, COCOON'2005, LNCS 3595, (2005), 671-679.

[23] T.-M. Wang and C.-C. Hsiao. On anti-magic labeling for graph products. Discrete Mathematics, 308(16), (2008), 3624-3633.

[24] Z. B. Yilma. Antimagic properties of graphs with large maximum degree. Journal of Graph Theory, 72(4), (2013), 367-373.

[25] Y. Zhang and X. Sun. The antimagicness of the Cartesian product of graphs. Theoretical Computer Science, 410(8-10), (2009), 727-735. 